\title{
Addressing psychosocial determinants and medication parameters in hypertension treatment models
}

\author{
Aayush Visaria $^{1,2} \cdot$ David Lo $^{2} \cdot$ Pranay Maniar ${ }^{2,3} \cdot$ Tina Dharamdasani $^{2}$ \\ Keywords Patient-centered medical home $\cdot$ Hypertension $\cdot$ Polypharmacy $\cdot$ Blood pressure variability
}

Received: 19 April 2020 / Revised: 19 April 2020 / Accepted: 7 June 2020 / Published online: 8 July 2020

(C) The Japanese Society of Hypertension 2020

\section{To the Editor:}

We read with great pleasure the article by John et al., titled "Effectiveness of a patient-centered medical home model of primary care versus standard care on blood pressure outcomes among hypertensive patients", which showed that patients in a patient-centered medical home $(\mathrm{PCMH})$ treatment model (known as WellNet) had greater reductions in systolic blood pressure (SBP) than matched controls who received standard care [1]. We applaud the authors' efforts to develop the WellNet PCMH model and determine its effectiveness; however, we would like to bring forth key considerations that may be necessary for a sustainable, comprehensive hypertension treatment model.

It has been well established that hypertension is the result of not only physiological factors but also psychosocial and environmental factors, including mental health, stress, socioeconomic status, education level, family support, and access to healthcare, among others [2-4]. While the authors noted that there were differences in behavioral factors such as smoking status between the treatment and comparison groups and possible sampling biases in education level and socioeconomic status that may compromise generalizability, it is also important to emphasize and specifically address mental health concerns. Undiagnosed depression, anxiety, and cognitive impairment are highly prevalent among elderly patients with several comorbidities [5]. Furthermore,

Aayush Visaria

aayush.visaria@rutgers.edu

1 Department of Medicine, Rutgers New Jersey Medical School, Newark, NY, USA

2 North American Disease Intervention, Rutgers University, New Brunswick, NJ, USA

3 New Jersey Institute of Technology, Newark, NJ, USA
Egede et al. showed that depression was associated with lower healthcare utilization and functional disability, both major barriers to effective care [5]. This may, in part, explain the decreased odds of blood pressure (BP) control seen with an increasing number of chronic conditions [1]. Including cognitive behavioral therapy as a routine part of the WellNet model, along with diet, exercise, motivational interviewing, and other standard considerations, may increase the efficacy of the model. Because this study was completed in a relatively affluent study population, it is difficult to ascertain the model's effectiveness in a population where environmental and psychosocial factors may play a larger role in hypertension treatment [6].

The study design for the treatment and comparison groups and the peculiar decrease in the mean number of medications in the comparison group may compromise the reliability of some of the results. First, although this study may be a secondary analysis, it would have been more appropriate to additionally match patients by SBP, as done in matching iterations $2-5$ in the original development of the cohort [7]. Second, the study population included individuals with hypertension who may not be taking any antihypertensive (anti-HTN) medications. Using the data given in Table 4 and presuming that the mean number of anti-HTN medications is only among those taking anti-HTN medications, we calculated that roughly 319 (91\%) and 2693 (88\%) of patients in the treatment and comparison groups, respectively, took anti-HTN medications. Nevertheless, to make accurate conclusions regarding medication use, it would be vital to know the proportion of hypertensive patients in each group taking BP medication, to classify the number of medications categorically by the number of anti-HTN classes, and to include the total number of medications taken. The total number of medications taken is important, as elderly patients with several comorbidities are at risk for polypharmacy and its associated negative outcomes [8]. The decrease in anti-HTN medications in the 
comparison group may be the result of physicians trying to decrease polypharmacy or optimize current medications, which may be beneficial long term. Whether the increase in BP medications seen in the treatment group is sustainable and without adverse effects over the course of several years remains to be seen. Third, several variables that would be necessary to assess the effectiveness of hypertension treatment other than absolute BP measures include kidney and cognitive parameters such as eGFR, albuminuria, and mental status exams. It is unclear why these parameters were not included in the analysis, as they are included in the original cohort design [7].

The significant differences in agents acting on the reninangiotensin-aldosterone system (C09) and antihypertensives (C02) may indicate differences in the severity or time of hypertension diagnosis between the treatment and comparison groups. The WellNet model may have differential effects on patients with a more recent diagnosis or lower severity of hypertension vs. others who have had hypertension and/or hypertension complications for many years. This is further supported by the study's result that every 1 $\mathrm{mmHg}$ increase in baseline SBP was associated with $15 \%$ lower odds of BP control over 12 months [1].

While there is a significant decrease in SBP in the treatment group, delineating the contribution of accessory activities such as smoking cessation and alcohol reduction in comparison to that of antihypertensive medication is vital for the development of improved models. Assessing the changes in smoking status, alcohol consumption, diet, exercise, and other parameters from the baseline visit to the 12-month follow-up would allow us to determine which aspects of the WellNet model were more effective than standard care.

As with any study examining changes in BP over time, it is increasingly important to consider physiologic visit-tovisit and measurement-to-measurement $\mathrm{BP}$ variability. Studies are increasingly showing that visit-to-visit and measurement-to-measurement BP variability are independent predictors of cardiovascular disease and mortality $[9,10]$. Furthermore, BP fluctuates throughout the day, so one-time BP readings may not be generalizable to a person's mean daily BP. Because treatment group patients had at least seven visits, it would be worthwhile to determine the significance of visit-to-visit BP variability within this group and the pattern of BP readings across the year rather than only at the endpoint.

Overall, we believe that this study makes an impactful contribution to the increasing literature on the effectiveness of the PCMH model and provides an example of a muchneeded, effective model for hypertension treatment and prevention. Further research should account for psychosocial determinants of health and provide comprehensive comparisons between treatment and control groups to allow for increased generalizability of results to the general Australian population and abroad.

\section{Compliance with ethical standards}

Conflict of interest The authors declare that they have no conflict of interest.

Ethical approval This study was exempt from Rutgers's Institutional Review Board approval.

Publisher's note Springer Nature remains neutral with regard to jurisdictional claims in published maps and institutional affiliations.

\section{References}

1. John JR, Tannous WK, Jones A. Effectiveness of a patientcentered medical home model of primary care versus standard care on blood pressure outcomes among hypertensive patients. Hypertens Res 2020. https://doi.org/10.1038/s41440-020-0431-3. [Epub ahead of print].

2. Basu S, Millett C. Social epidemiology of hypertension in middleincome countries: determinants of prevalence, diagnosis, treatment, and control in the WHO SAGE study. Hypertension. 2013;62:18-26.

3. Nabi H, Chastang JF, Lefèvre T, Dugravot A, Melchior M, Marmot MG, et al. Trajectories of depressive episodes and hypertension over 24 years: the Whitehall II prospective cohort study. Hypertension. 2011;57:710-6.

4. Durrani S, Irvine J, Nolan RP. Psychosocial determinants of health behaviour change in an e-counseling intervention for hypertension. Int J Hypertens. 2011;2012:1-5.

5. Egede LE. Major depression in individuals with chronic medical disorders: prevalence, correlates and association with health resource utilization, lost productivity and functional disability. Gen Hosp Psychiatry. 2007;29:409-16.

6. Visaria A, Dharamdasani T. Necessary complexities when standardizing hypertension protocols in India. Indian Heart J. 2019. https://doi.org/10.1016/j.ihj.2020.01.002. [Epub ahead of print].

7. John JR, Jones A, Neville AM, Ghassempour S, Girosi F, Tannous WK. Cohort profile: effectiveness of a 12-Month Patient Centred Medical Home Model versus standard care for chronic disease management among primary care patients in Sydney, Australia. Int J Environ Res Public Health. 2020;17:2164-85.

8. Davies LE, Spiers G, Kingston A, Todd A, Adamson J, Hanratty B. Adverse outcomes of polypharmacy in older people: systematic review of reviews. J. Am. Med. Dir Assoc. 2020;21:181-7.

9. Eguchi K, Hoshide S, Schwartz JE, Shimada K, Kario K. Visit-tovisit and ambulatory blood pressure variability as predictors of incident cardiovascular events in patients with hypertension. Am J Hypertens. 2012;25:962-8.

10. Visaria A, Amorosa L. Measurement-to-measurement blood pressure variability as a predictor of cardiovascular disease outcomes. J Am Coll Cardiol. 2020;75:1947. 